\title{
Intubación traqueal retrógrada en paciente con anquilosis de la articulación temporomaxilar
}

Retrograde tracheal intubation in a patient with temporomaxillary joint ankylosis

\author{
Freddy Espinoza Mendoza ${ }^{1,2}$, Jesús Alvarado Rivera ${ }^{3}$, María Elena León Meléndez \\ Departamento de Anestesiología y Centro Quirúrgico, Hospital Nacional Daniel A. Carrión, Callao, Perú. \\ ${ }^{1}$ Anestesiólogo, Hospital Nacional Daniel A. Carrión. \\ ${ }^{2}$ Editor en Jefe, Revista Actas Peruanas de Anestesiología. \\ ${ }^{3}$ Residente de anestesiología, Universidad Nacional Mayor de San Marcos.
}

\begin{abstract}
Resumen
La vía aérea difícil es uno de los mayores retos para el anestesiólogo y la técnica de intubación traqueal retrógrada es considerada una opción válida pero poco utilizada cuando se presenta una intubación difícil. Se comunica el caso de un paciente de 59 años, programado para amputación supracondilea de pierna, con antecedente de anquilosis de la articulación temporomaxilar secundaria a fractura de la rama ascendente del maxilar inferior. Al examen físico presentaba limitación marcada de la apertura bucal. La cirugía se programó bajo anestesia general y, ante la imposibilidad de una intubación convencional, se decidió realizar una intubación traqueal retrógrada. El procedimiento se llevó a cabo con éxito e hizo posible la intervención quirúrgica.
\end{abstract}

Palabras clave: Intubación intratraqueal, anestesia intratraqueal, articulación temporomandibular.

Abstract

Difficult airway is one of the biggest challenges for the anesthesiologist and the technique of retrograde tracheal intubation is considered an option but subutilized when there is difficult in intubation. We report the case of a 59 year-old patient scheduled for supracondylar amputation of his leg, with a history of temporomandibular joint ankylosis secondary to fracture of the mandibular ramus. Physical examination showed marked limitation of mouth opening. Surgery was scheduled under general anesthesia and because the impossibility of conventional intubation retrograde tracheal intubation was performed. The procedure was carried out successfully and surgery was possible.

Key words: Intubation/intratracheal, anesthesia/intratracheal, temporomandibular joint.

An Fac med. 2012;73(3):251-4

\section{INTRODUCCIÓN}

El manejo de la vía aérea difícil es uno de los mayores retos para el anestesiólogo. La Sociedad Americana de Anestesiólogos (ASA) la define como la situación clínica en la cual un anestesiólogo convencionalmente entrenado experimenta dificultad con la ventilación de la vía aérea superior con máscara facial, dificultad para la intubación traqueal o ambos (1). Diversas sociedades de anestesiología, instituciones docentes y hospitales han elaborado guías y protocolos para su manejo, y en la mayoría de ellas se menciona a la técnica de la intubación traqueal retrógrada (ITR) como una alternativa, tanto en cirugías electivas como de emergencia.
La ITR, aunque es una técnica mínimamente invasiva y fácil de realizar por manos experimentadas, es poco utilizada ${ }^{(2)}$. Fue descrita por primera vez por Butler ${ }^{(3)}$, en 1960, al deslizar un tubo endotraqueal sobre un catéter $16 \mathrm{~F}$, el cual había sido pasado, con su guía en el interior, por una traqueostomía preexistente hasta la boca. La principal ventaja de esta técnica sobre otras más comunes de intubación anterógrada es que no requiere de equipo sofisticado ni de la visualización de la glotis $(4,5)$.

A continuación presentamos un caso de aplicación de la técnica de ITR en un paciente con limitación de la apertura bucal, una de las indicaciones que tiene este procedimiento ${ }^{(4)}$.

\section{REPORTE DE CASO}

Paciente de sexo masculino, 59 años de edad y en abandono social, programado para amputación supracondílea de pierna derecha por osteomielitis crónica. Presentaba como antecedente anquilosis de la articulación temporomaxilar derecha secundaria a fractura, por accidente automovilístico, de la rama ascendente del maxilar inferior hacía 35 años, sin tratamiento médico o quirúrgico.

En la evaluación preanestésica refería consumo antiguo de alcohol y tabaco, y hasta hace 3 años de marihuana. Al examen físico se evidenció paciente en regular estado general y mal estado 
de nutrición $\left(17,63 \mathrm{~kg} / \mathrm{m}^{2}\right.$ de índice de masa corporal). En la valoración de la vía aérea, la clasificación de Mallampati no podía ser realizada por la marcada limitación de la apertura bucal menor de $2 \mathrm{~cm}$ (figuras 1 y 2 ); la distancia tiromentoniana era mayor de $6 \mathrm{~cm}$ y los dientes se encontraban en mal estado general. En los exámenes auxiliares, la hemoglobina era de $9 \mathrm{mg} / \mathrm{dL}$ y el riesgo quirúrgico cardiovascular II-III. Se le catalogó como intubación difícil y ASA II.

Ante la negativa del paciente para administrársele anestesia regional, se decidió la programación de la amputación bajo anestesia general balanceada y, ante la imposibilidad de realizar una intubación convencional, una intubación nasotraqueal bajo sedación y con apoyo del fibrobroncoscopio del servicio de neumología. Sin embargo, la cirugía se postergó en varias ocasiones por falta de coordinación entre traumatología, anestesiología y neumología. Finalmente, cuando se ingresó a sala de operaciones se planteó la posibilidad de realizar una ITR bajo sedación, la cual fue autorizada por el paciente. Ante cualquier eventualidad, se contaba con el apoyo del fibrobroncoscopio.

El paciente ingresó a sala de operaciones con presión arterial de 105/80 $\mathrm{mm} \mathrm{Hg}$, frecuencia cardíaca de 83 latidos por minuto y saturación de oxígeno $96 \%$. Previa oxigenación a través de máscara de mediana concentración a flujo de $6 \mathrm{~L} / \mathrm{min}$ y la administración de fentanilo $100 \mu \mathrm{g}$ y lidocaína $40 \mathrm{mg}$ endovenosos, se realizó el bloqueo del nervio laríngeo superior con lidocaína $2 \%, 2 \mathrm{~mL}$ por cada rama, y del nervio laríngeo recurrente con lidocaína $2 \%$, $2 \mathrm{~mL}$.

Luego de esperar un tiempo prudente para que el anestésico local hiciera efecto y con el paciente bajo sedación Ramsay 2, se procedió a la perforación de la membrana cricotiroidea con un catéter endovenoso periférico $16 \mathrm{G}$ conectado a una jeringa de $10 \mathrm{~mL}$ que realizaba presión negativa, avanzando hasta localizar el espacio traqueal. El

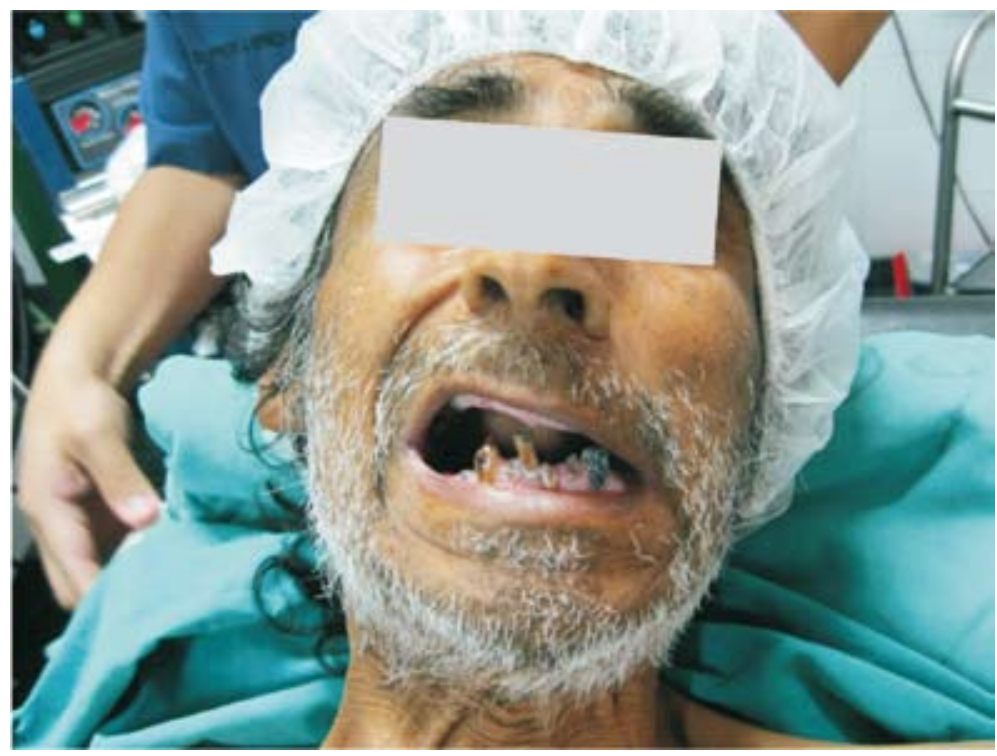

Figura 1. Vista de frente del paciente, donde a la apertura máxima no es posible realizar la clasificación de Mallampati. Obsérvese también el mal estado de los dientes.

procedimiento provocó ansiedad y reflejo de tos en el paciente, produciendo movilización del catéter, por lo que se decidió retirarlo. Luego de recanalizar la tráquea en segundo intento, se administró por el mismo $1 \mathrm{~mL}$ de lidocaína $2 \%$.

Una vez que se consiguió inhibir el reflejo de tos, se procedió a pasar un ca- téter peridural (Perifix ${ }^{\circledR}$ de B. Braun) 18 $\mathrm{G}$, hasta llegar a orofaringe, momento en que el paciente colaboró expulsando la punta de catéter por la boca. Se procedió a pasar un tubo endotraqueal D.I. 7,0 a través de la guía hasta la epiglotis, momento en el que el paciente se agitó y empezó a toser, por lo que se

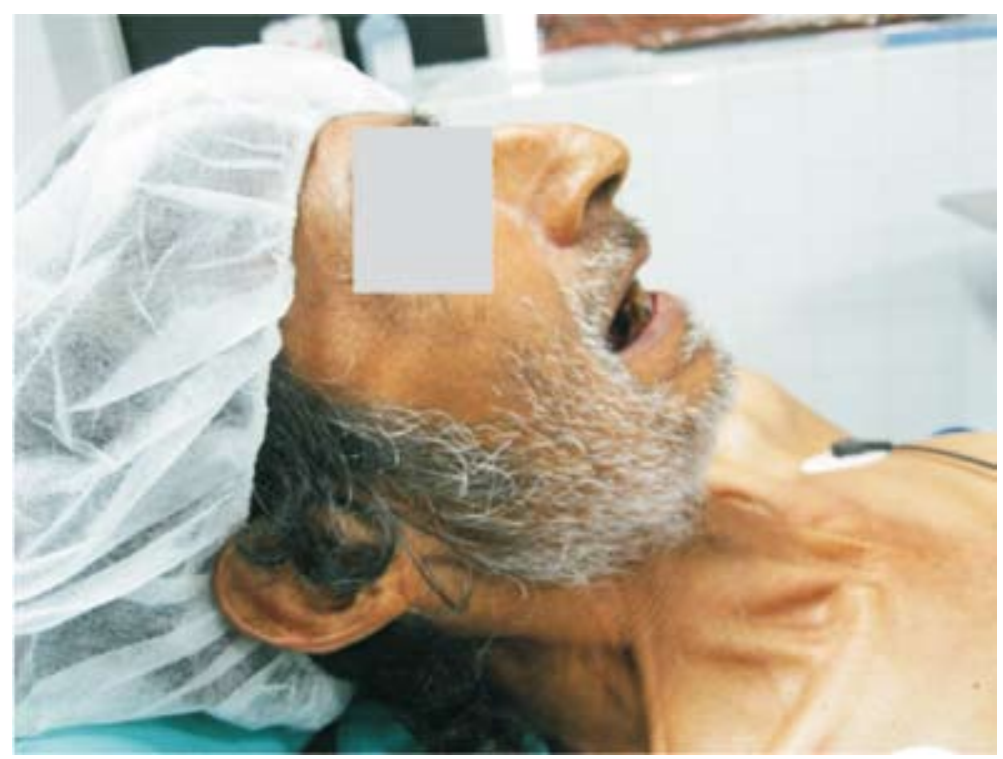

Figura 2. Vista de perfil del paciente, donde se puede apreciar la limitada apertura bucal, menor de $2 \mathrm{~cm}$. 


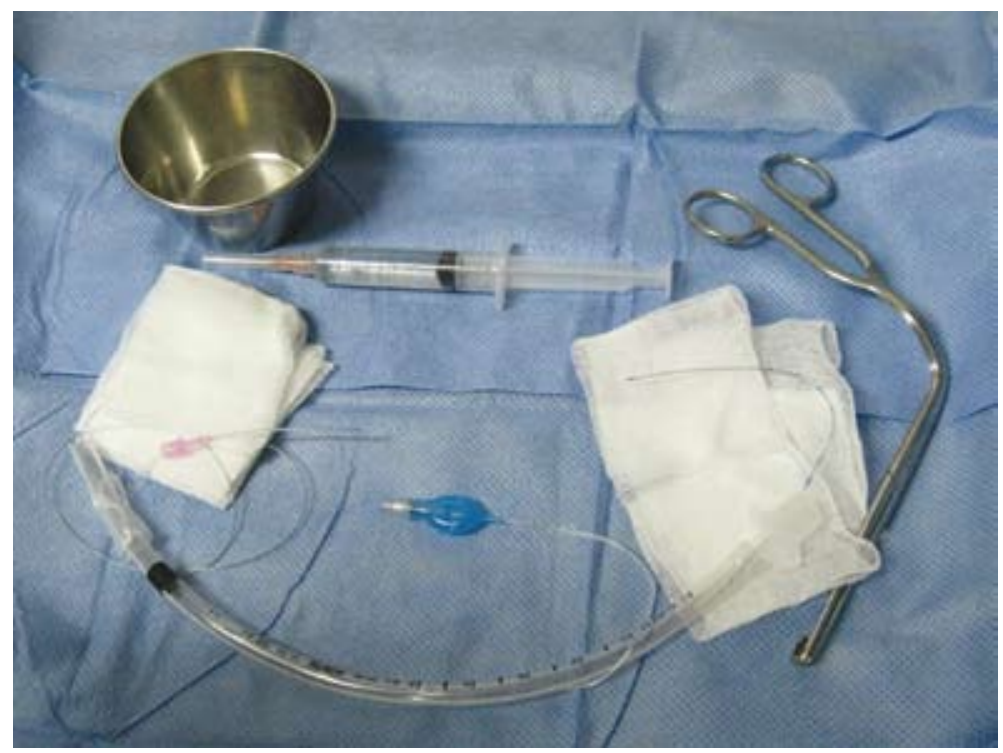

Figura 3. Material utilizado para la realización de la intubación traqueal retrógrada.

administró adicionalmente midazolam $1 \mathrm{mg}$, lidocaína $40 \mathrm{mg}$ y propofol 60 $\mathrm{mg}$, vía endovenosa, consiguiendo, con paciente dormido, intubación al tercer intento.

Se constató colocación de tubo endotraqueal con capnografía y auscultación de campos pulmonares. Se procedió a la administración de rocuronio 10 $\mathrm{mg}$, infusión de remifentanilo $0,2 \mu \mathrm{g} /$ $\mathrm{kg} / \mathrm{min}$ y sevoflurano $1,5 \%$, para luego dar paso a la cirugía. El procedimiento duró aproximadamente una hora, sin intercurrencias. Una vez concluida la operación, se descontinuó el remifentanilo y el sevoflurano y se procedió a la extubación del paciente despierto, pasando a la unidad de recuperación postanestésica.

\section{DISCUSIÓN}

Para asegurar el control de la vía aérea de un paciente con limitación de la apertura bucal, los diferentes algoritmos ofrecen múltiples alternativas que incluyen desde la no invasiva, como la intubación nasal a ciegas, hasta la opción quirúrgica de una traqueostomía. En este caso se decidió por una ITR, pues dado que la apertura bucal permi- tía al paciente ingerir alimentos, debía permitirle poder colaborar con la salida del catéter por la boca, tal como sucedió. Esto obvió una de las principales dificultades que tiene esta técnica, la cual es la recuperación de la guía retrógrada a través de la vía aérea superior ${ }^{(6)}$.

Aunque la ITR es un procedimiento relativamente rápido y eficaz con el entrenamiento adecuado, no es una técnica que esté exenta de falla. Entre las causas, se ha analizado las relacionadas al sitio de punción, la distancia entre la membrana cricoides y las cuerdas vocales y el uso de diferentes guías y catéteres para la correcta inserción del tubo a través de la glotis. Esto ha generado el desarrollo de variantes de la técnica ${ }^{(6-10)}$, a lo largo de los años, a fin de adaptarla a diferentes situaciones clínicas así como a los equipos disponibles.

En el caso que presentamos se utilizó para la realización del procedimiento un catéter endovenoso periférico $16 \mathrm{G}$ y un catéter peridural $18 \mathrm{G}$ (figura 3). Se ha descrito también el uso de agujas peridurales y guía de catéter endovenoso central ${ }^{(4)}$; sin embargo, lo ideal es el uso de equipos diseñados especialmente para tal fin, como son el set de intubación retrógrada de Cook (Cook Critical Care) ${ }^{(5)}$. En nuestro hospital, como sucede en muchos de nuestra región, no contamos ni con la logística ni los recursos económicos necesarios para poder adquirir estos dispositivos, los cuales incluso están considerados por la ASA como indispensables en los coches para el manejo de la vía aérea difícil (1).

Las dificultades presentadas al momento de abordar el espacio traqueal pudieron deberse a la poca dosis utilizada en el bloqueo del nervio laríngeo. Las técnicas de anestesia regional sugieren el uso de $5 \mathrm{~mL}$ de lidocaína $2 \%$, o incluso más, por cada rama; sin embargo, debido al estado nutricional disminuido del paciente, que determinaba un escaso tejido subcutáneo en la zona, decidimos administrar solo $2 \mathrm{~mL}$ por lado, lo cual aparentemente fue insuficiente.

El otro inconveniente presentado fue al deslizar el tubo endotraqueal sobre el catéter peridural. El hecho de que el paciente se agitara y se desencadenara el reflejo de tos cuando el tubo llegó a la glotis, es otro indicativo de que no se había conseguido un bloqueo adecuado de los nervios laríngeos. Para evitar este problema, muchos autores sugieren realizar el procedimiento completo bajo anestesia general ${ }^{(6,7)}$. El temor al margen de error de esta técnica, sumado a la poca experiencia que teníamos de ella, fue lo que motivó que se insistiera en intentarlo solo bajo sedación, pese a contar con el respaldo del fibrobroncoscopio por cualquier contingencia. Únicamente fue posible la intubación del paciente tras tres intentos y una vez que este fue dormido con $60 \mathrm{mg}$ de propofol endovenoso.

No se presentó alguna de las complicaciones descritas, ni las que tienen que ver con el uso de material que no fue designado para este uso, como el catéter endovenoso y el catéter peridural, ni las propias del procedimiento, como enfisema, infección, edema o dolor laríngeo. Los informes de inconvenientes de una ITR son en realidad escasos y 
disminuyen en relación inversa a la experiencia del operador, de tal manera que se puede considerar a esta como una técnica segura ${ }^{(9)}$.

La ITR no requiere de gran entrenamiento y puede ser aprendida rápidamente. Sin embargo, no es considerada dentro de los objetivos de nuestras instituciones formadoras de anestesiólogos. Esto difiere de otras realidades, como la del Reino Unido, donde esta técnica está incluida en los planes curriculares del Colegio Real de Anestesiólogos ${ }^{(4)}$. Si resultara difícil, por cuestiones éticas, el adiestramiento con pacientes en la práctica clínica, puede disponerse del uso de cadáveres, lo que ha mostrado tener buenos resultados. Luego del entrenamiento en cadáveres, la proporción de residentes que comunicaron que usarían esta técnica se incrementó de 6 a $67 \%$ y aquellos que señalaron que podían realizar el procedimiento correctamente se incrementó de 28 a $83 \%{ }^{(11)}$.

En conclusión, la ITR es una alternativa fácil y que no requiere de equi- po sofisticado para el manejo de la vía aérea difícil, pese a lo cual no es muy utilizada entre los anestesiólogos. El residente de la especialidad debería recibir entrenamiento continuo para el dominio de esta técnica. Los servicios de anestesiología deben contar, en el coche de manejo de vía aérea difícil, con el material necesario para poder llevar a cabo esta técnica.

\section{REFERENCIAS BIBLIOGRÁFICAS}

1. American Society of Anesthesiologists Task Force on Management of the Difficult Airway. Practice guidelines for management of the difficult airway: an updated report by the American Society of Anesthesiologists Task Force on Management of the Difficult Airway. Anesthesiology. 2003;98:1269-77.

2. Benumof JL. Mamagement of the difficult adult airway. With special emphasis on awake tracheal intubation. Anesthesiology. 1991;75:1087-110.

3. Butler FS, Cirillo AA. Retrograde tracheal intubation. Anesth Analg. 1960;39:333-8.

4. Dhara SS. Retrograde tracheal intubation. Anaesthesia. 2009;64:1094-104.

5. Langeron O, Birenbaum A, Amour J. Airway management in trauma. Minerva Anestesiol. 2009;75:307-11.

6. Bhattacharya P, Biswas BK, Baniwal S. Retrieval of a retrograde catheter using suction, in patients who cannot open their mouths. $\mathrm{Br} J$ Anaesth. 2004;92:888-90.
7. Mahajan R, Sandhya X, Chari P. An alternative technique for retrograde intubation. Anaesthesia. 2001;56:1207-8.

8. Harrison WL, Bertrand ML, Andeweg SK, Clark JA. Retrograde intubation around an in situ combitube: a difficult airway management strategy. Anesthesiology. 2005;102:1061-2.

9. Lenfant F, Benkhadra M, Trouilloud P, Freysz M. Comparison of two techniques for retrograde tracheal intubation in human fresh cadavers. Anesthesiology. 2006;104:48-51.

10. Marciniak D, Smith CE. Emergent retrograde tracheal intubation with a gum-elastic bougie in a trauma patient. Anesth Analg. 2007;105:1720-1.

11. Hatton KW, Price S, Craig L, Grider JS. Educating anesthesiology residents to perform percutaneous cricothyrotomy, retrograde intubation, and fiberoptic bronchoscopy using preserved cadavers. Anesth Analg. 2006;103:1205-8.

Artículo recibido el 29 de noviembre de 2011 y aceptado para publicación el 16 de mayo de 2012.

Fuente de financiamiento: ninguna.

Conflictos de interés: ninguno.

Correspondencia:

Dr. Freddy Espinoza Mendoza

Dirección: Jr. Padre Urraca 265-A Urb. Maranga, Lima 32 - Perú

Teléfono: 578-1330 Fax: 460-3499

Correo electrónico: fespinozam@hotmail.com 